\title{
Ameliorative Effect of Falcaria vulgaris on Nicotine-induced Injury on the Hippocampus Dentate Gyrus Region of Rats
}

\author{
Shiva Roshankhah', Amir Abdolmaleki', Cyrus Jalili' ${ }^{2}$, Mohammad Reza Salahshoor ${ }^{1, *}$ \\ 'Department of Anatomical Sciences, Medical School, Kermanshah University of Medical Sciences, Daneshgah Ave., Taghbostan, Kermanshah, IRAN. \\ ${ }^{2}$ Medical Biology Research Center, Department of Anatomical Sciences, Kermanshah University of Medical Sciences, Daneshgah Ave., Taghbostan, Kermanshah, IRAN.
}

\begin{abstract}
Objectives: The dentate gyrus (DG) is a part of a brain region known as hippocampus. Falcaria vulgaris (FV) is a vegetable that contains antioxidant ingredients. Nicotine is one of the main risk factors in functional disorders of various organ systems. This study was designed to evaluate the therapeutic effects of $F V$ against nicotine induced injury on the hippocampus DG region of rats. Methods: In this study, 64 male rats were randomly assigned to 8 groups: Sham group, nicotine group $(0.5 \mathrm{mg} / \mathrm{kg})$, FV groups $(50,100$ and $150 \mathrm{mg} / \mathrm{kg})$ and nicotine + FV groups. Treatments were administered intraperitoneally daily for 28 days. Ferric Reducing Antioxidant Power (FRAP) method was applied to determine the total antioxidant capacity. The number of dendritic spines was investigated by Golgi technique. Cresyl violet method was used to determine the number of neurons in DG region. Also, Griess technique was used to determine serum nitrite oxide level. Results: Nicotine administration increased significantly nitrite oxide level and total antioxidant capacity and a decreased number of neuronal
\end{abstract}

dendritic spines and neurons compared to the sham group $(P<0.05)$. In the FV and nicotine + FV groups, in all dosages, the number of neurons and neuronal dendritic spines increased significantly while nitrite oxide level and total antioxidant capacity decreased compared to the nicotine group ( $P$ $<0.05)$. Conclusion: It seems that FV administration improves DG region injury in rates because of nicotine.

Key words: Falcaria vulgaris, Dentate gyrus, Nicotine, Hippocampus, Rats.

Correspondence

Dr. Mohammad Reza Salahshoor,

Department of Anatomical Sciences, Medical School, Kermanshah University of Medical Sciences, Kermanshah, IRAN.

Phone no: +98 09188360349

Email: reza.salahshoor@yahoo.com

DOI: 10.5330/ijpi.2019.4.37

\section{INTRODUCTION}

The FV is a vegetable belonging to the Apiaceae (Umbelliferae) family that grows in the west and southwest of Iran as an annual wild plant. ${ }^{1} \mathrm{FV}$ has anti-oxidant properties. ${ }^{2}$ This plant has a stem and its height is $30 \mathrm{~cm}$ on average. In the west of Iran, this plant has been traditionally used to treat skin ulcers, gastric disorders, including gastric ulcer, liver disease, kidney stones and bladder. ${ }^{3}$ Phytochemical studies on this medicinal plant have shown the presence of Tannins and Saponin. ${ }^{4}$ This plant contains Vitamin C, phytosterol, protein and starchy materials and is a rich source of tannins and ascorbic acid. Similar to antibiotics, it can be used for the treatment of skin ulcers. ${ }^{5}$ Tobacco consumption has declined dramatically in industrialized countries over the past three decades due to raising awareness of the health risks and effective control policies, while it has increased in developing countries over the same period. ${ }^{6}$ The overall nicotine contented of tobacco and percentage nicotine is an average of $10.2 \mathrm{mg} .{ }^{7}$ Nicotine spreads rapidly in brain tissue through pulmonary circulation within 10-20 sec and attaches to the Nicotinic Acetylcholine Receptors (nAChRs). ${ }^{8}$ Nicotine rapidly passes through the blood-brain barrier and stimulates the mesolimbic dopamine system. This substance can regulate brain neurotransmitters, including catecholamine and serotonin A. ${ }^{9}$ However, nicotine can induce oxidative stress in some organs including the brain. Pathologic changes associated with neuronal apoptosis have been reported due to the use of nicotine. ${ }^{10}$ Also, nicotine can induce increased oxidative stress and neuronal apoptosis, destroy DNA, produce reactive oxygen species and increase the production of lipid peroxidation. ${ }^{11,12}$ This compound seems to activate the areas of the brain that play an important role in drug addiction and learning process. Among the brain areas greatly affected by nicotine is mesocorticolimbic region that contains nucleus accumbens, ventral tegmental area, the hip- pocampus and the amygdala. In this region, the amygdala and hippocampus have structures that play an important role in the formation of long-term memory and their function is associated with stimulation reward system. ${ }^{13}$ Hippocampus is a part of the limbic system and seems to be essential in the formation of different types of learning and memory. ${ }^{14}$ The dentate gyrus is a part of hippocampus and contributes in formation of new episodic memories. ${ }^{15}$ Considering the effects of nicotine toxicity on the brain and the therapeutic properties of FV, the present study was designed and conducted to investigate the effects of FV on nicotineinduced toxicity in the hippocampus DG region of male rats.

\section{MATERIALS AND METHODS}

\section{Animals}

In this experimental study, 64 male Wistar rats, weighing 220-250 g were purchased from the Pasteur Institute and transferred to the animal house in medical school. During the study, the animals were kept under standard conditions (i.e., $12 \mathrm{hr}$ light $/ 12 \mathrm{hr}$ dark and $22 \pm 2^{\circ} \mathrm{C}$ ), humidity of $50 \%-60 \%$, in special cages and on a straw bed. Water and food were freely available to the animals. Standard food was including plate and treated municipal water was used to feed the animals. All investigations conformed to the ethical and human principles of research and were approved by the Ethics Committee (ethics certificate No. 97601). ${ }^{16}$

\section{Extract preparation}

FV plant was obtained from a local store (time to pick and buy this plant in the spring) and its impurities were removed. After endorsement by a botanist, the plant was cleaned. The leaves and stems were desiccated in 
shadow for 5 days and ground using a grinder. Next, $100 \mathrm{~g}$ of the powder was added to $70 \%$ ethanol. The acquired solution was reserved in a warm water bath $\left(36^{\circ} \mathrm{C}\right)$ under dark condition. Thereafter, the solution was progressively poured on Buchner funnel filter paper and cleaned by a vacuum pump. It was then transferred to a rotary device to obtain the extra solvent. The isolation process continued until the concentrated extract was obtained. The extract was dissolved in distilled water and administered intraperitoneally per a kilogram of animal's weight. It was sterilized after double filtration through a $0.2-\mu \mathrm{m}$ filter. ${ }^{2}$

\section{Study groups and treatment of animals}

Vial of nicotine (Sigma, USA) with a dose of $0.5 \mathrm{mg} / \mathrm{kg}$ of body weight was dissolved in $0.9 \%$ normal saline solution. A total of 64 male rats were randomly divided into 8 groups and 8 rats were placed in each group. The first group was sham group, which received normal saline through intraperitoneal injection equivalent to the amount of experimental groups. In the second nicotine group, each animal received 0.5 $\mathrm{mg} / \mathrm{kg}$ single dose course of nicotine, intraperitoneally and the solvent of nicotine was normal saline. ${ }^{10}$ The third to fifth groups included the FV groups, in which each animal respectively received 50, 100 and $150 \mathrm{mg} /$ $\mathrm{kg}$ of FV intraperitoneally for 28 days at 10 a.m. ${ }^{1,2,4}$ The sixth to eighth groups include nicotine $+\mathrm{FV}$ groups, in which each animal received a single dose of $0.5 \mathrm{mg} / \mathrm{kg}$ nicotine in order to induce damage and then respectively received 50, 100 and $150 \mathrm{mg} / \mathrm{kg}$ of FV intraperitoneally for 28 day at 10 a.m.

\section{Transcardiac perfusion}

The transcardiac method was used for fixation. In this process, $24 \mathrm{hr}$ after the last injection of the drug, animals were anesthetized with Ketamine $(100 \mathrm{mg} / \mathrm{kg})$ and Xylazine $(10 \mathrm{mg} / \mathrm{kg})$. The chest was opened in the midline and the apex of the left ventricle was pierced after the completion of thoracotomy. Next, a glass cannula of $1 \mathrm{~mm}$ diameter was inserted and fixed in the ascending aorta. The pericardium and the right ventricle were cut. The left ventricle pathway was cut and the ascending aorta was connected to a plastic tube by the glass cannula and descending aorta was clamped right above the diaphragm. The cannula linked to the normal saline solution was implanted into the aorta through making an incision in the left ventricle. The descending aorta was fastened and after washing the brain, the solution was removed through the incision made in the right atrium. Formalin $5 \%$ and buffer phosphate $7 \%$ were inoculated into the brain by the cannula and the brain were fixed in 15 min. After perfusion, the brains were separated from the skull and stored in the same perfusion solution for 3 days. ${ }^{14}$

\section{Golgi methods}

The Golgi method was used to observe neuron dendrites in the hippocampus DG region. This method was applied using potassium dichromate followed by silver nitrate. After brain fixation, tissue blocks were put inside $3 \%$ potassium dichromate solution for $48 \mathrm{~h}$ in a dark environment. The blocks were washed in $0.75 \%$ silver nitrate solution and were put inside the solution for $72 \mathrm{hr}$. The tissues were washed in $1 \%$ silver nitrate solution. Then, tissue processing, counting dehydration, clearing and embedding were performed. Microscopic sections $(5 \mu \mathrm{m})$ were prepared and examined morphologically. ${ }^{15}$

\section{Cresyl violet method}

The Cresyl violet staining method was used to determine the number of live cells in the hippocampus DG region. For this purpose, eight rat heads from each group (8 groups) and 5 slides from each rat were taken to be stained. Subsequently, after producing $5 \mu \mathrm{m}$ cuts by microtome and performing tissue processing, the left hemispheres were stained using
Cresyl violet staining technique. In brief, the slips were stable again (10 $\min$ ) in $4 \%$ paraformaldehyde solution. Slide was immersed in $70 \%$ (5 $\mathrm{min}$ ) and $100 \%(15 \mathrm{~min})$ ethanol and in xylene for $20 \mathrm{~min}$. They were then immersed back through the ethanol descent concentrations. They were stained for $5 \mathrm{~min}$ in filtered Cresyl violet solution and then washed in distilled water. The slides were then dehydrated again in ethanol. They were located in xylene for another $10 \mathrm{~min}$ and then cover slipped. After preparing the photo, the number of cells was counted in one square millimeter $\left(\mathrm{mm}^{2}\right)$. In the slides stained by means of Cresyl violet technique, the round cells without peak nose were considered as live cells. ${ }^{14}$

\section{Dendritic thorns}

The dendritic thorn count was made via microscopic examination with an optical microscope and Motic software and Image tool IT (version 3) software. In the slides stained through Golgi staining technique, neurons entirely stained with cell bodies in the central part of the tissue slices distant from the surrounding stained neurons were included. The dendritic tree of pyramidal neurons was demonstrated through camera lucida at $750 \times$ magnification and the dendritic exclusion order from the cell body was used for counting the dendritic pieces. ${ }^{14}$

\section{Griess technique}

Nitrite oxide, measurement by Griess assay using microplate technique. Through this process, zinc sulfate powder $(6 \mathrm{mg})$ was mixed with serum samples $(400 \mu \mathrm{l})$ and vortexed for $1 \mathrm{~min}$. The samples were centrifuged at $4^{\circ} \mathrm{C}$ for $10 \mathrm{~min}$ at $12,000 \mathrm{rpm}$ and supernatant was used to measure the nitrite oxide. Briefly, $50 \mu \mathrm{l}$ of sample was added to $100 \mu \mathrm{l}$ of Griess reagent (Sigma; USA) and the reaction mixture was incubated for about $30 \mathrm{~min}$ at room temperature. The Optical Density (OD) of the sample was measured by an ELISA reader (Hyperion; USA) at a wavelength of $540 \mathrm{~nm}$ according to manufacturer protocol. ${ }^{16}$

\section{FRAP method}

FRAP method was used in order to the measure total antioxidant capacity of the serum (Abcam, ab234626). Before transcardiac method, venipuncture from the animals' hearts (right ventricle) was done using a $5 \mathrm{cc}$ syringe. The blood sample was incubated for $15 \mathrm{~min}$ at $37^{\circ} \mathrm{C}$ to clot. Then the clotted blood was then centrifuged for $15 \mathrm{~min}$ at $3000 \mathrm{rpm}$ until the serum was separated. The separated serum was stored at the temperature of $-70^{\circ} \mathrm{C}$ until antioxidant capacity level was measured. In this technique, the ability of the plasma to reinstate ferric ions was measured. This process required a great quantity of $\mathrm{Fe}^{\mathrm{III}}$. A blue stain was formed when the compound of $\mathrm{Fe}^{\mathrm{III}}$-TPTZ in acidic $\mathrm{pH}$ returned to $\mathrm{Fe}^{\mathrm{II}}$ and absorption at the maximum wavelength of $593 \mathrm{~nm}$. The only factor defining the speed of the $\mathrm{Fe}^{\mathrm{II}}$-TPTZ and the blue color was the vitalizing power of the sample. Total antioxidant capacity values were strategized by means of the standard curve with diverse concentrations of iron sulfate. ${ }^{17}$

\section{Statistical analysis}

After extracting the information, Kolmogorov-Smirnov test was first conducted to confirm the data compliance of the normal distribution. The One way analysis of variance (one-way ANOVA) was used for statistical analysis and Tukey post hoc test was used to determine the difference between the groups. SPSS 16 was used for data analysis. The obtained results were expressed as mean \pm standard error and $p<0.05$ was considered statistically significant.

\section{RESULTS}

\section{Neurons number}

The results of a mean number of neurons in the DG showed a significant decrease in nicotine group compared to the sham group $(P<0.05)$. The 


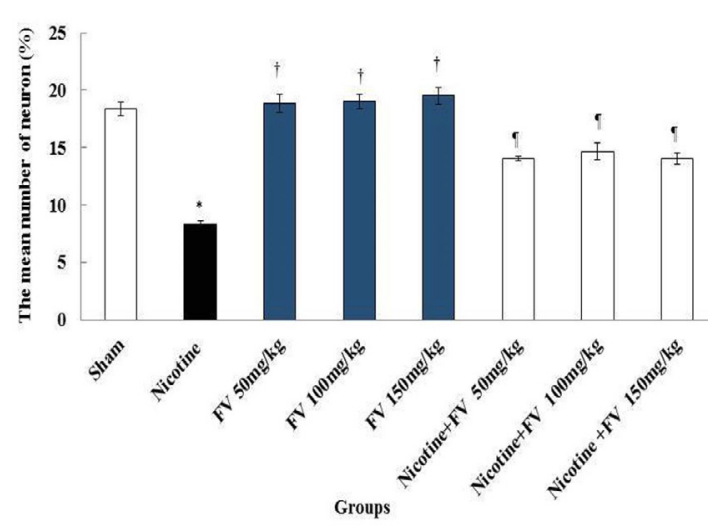

Figure 1: Effect of nicotine, FV and nicotine + FV administration on the mean number of neurons in the DG region; *Significant difference compared to the sham group $(P<0.01)$; $†$ Significant difference compared to the nicotine group $(P<0.01)$; ISignificant difference compared to the nicotine and sham groups $(P<0.01)$. FV: Falcaria vulgaris, DG: Dentate gyrus.

mean number of neurons was not significant in all FV groups compared to the sham group $(P>0.05)$. Also, the mean of neurons increases significantly in all FV and nicotine $+\mathrm{FV}$ groups compared to the nicotine group $(P<0.05)$ [Figures 1 and 2 ].

\section{Dendritic spines}

The mean number of neuronal dendritic spines in experimental groups showed a significant decrease between the sham group and nicotine group $(P<0.05)$. Also, the mean number of neuronal dendritic spines was not significant in all FV groups compared to the sham group $(P>$ $0.05)$. Further, at the FV and nicotine + FV groups, the mean number of neuronal dendritic spines increases significantly in all treated groups compared to the nicotine group $(P<0.05)$ [Figures 3 and 4 ].

\section{Nitrite oxide}

The results of blood serum nitrite oxide measurement showed a significant increase in nicotine group compared to the sham group $(P<0.05)$. The mean nitrite oxide in the blood serum was not significant in all FV groups compared to the sham group $(P>0.05)$. Also, the mean of nitrite oxide in blood serum declined significantly in all FV and nicotine $+\mathrm{FV}$ groups compared to the nicotine group $(P<0.05)$ [Figures 5].

\section{Total antioxidant capacity}

The results displayed that the total antioxidant capacity serum level reduced significantly in the nicotine group compared to the sham group ( $P$ $<0.05)$. The total antioxidant capacity level enhanced significantly in all $\mathrm{FV}$ and nicotine $+\mathrm{FV}$ groups compared to the nicotine group $(P<0.05)$ [Figures 6].

\section{DISCUSSION}

Temporal lobe and hippocampal organization are involved in transferring short-term memory to long-term memory. ${ }^{18}$ Nicotine produces many implications and side effects by influencing the central and peripheral nervous system. ${ }^{7,10}$ The present study was aimed to investigate the effects of FV on nicotine-induced disorders in the hippocampus DG region. The number of neurons and dendritic thorns decreased significantly in the nicotine group comparted to the sham group. In nicotine + FV groups, there was a significant increase in the mean of neuron numbers and dendritic thorns compared to the nicotine group. The results may indicate the control of apoptosis and neurodegeneration by administering different doses of FV. The results may indicate the control

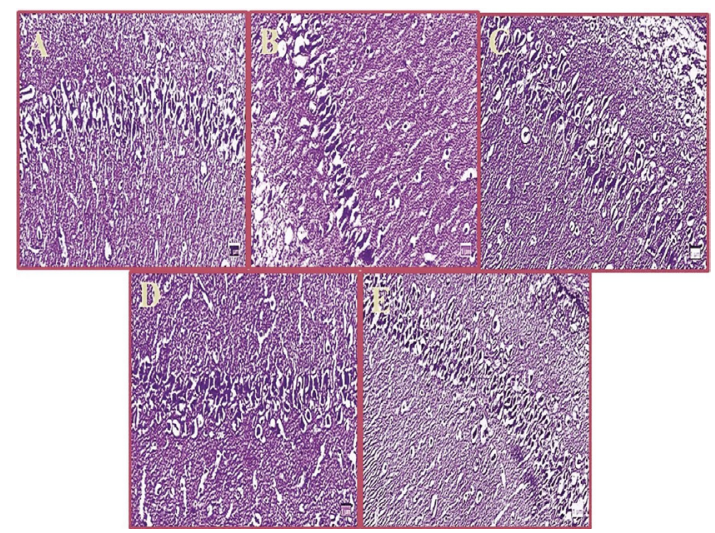

Figure 2: Microscopic images of DG region in male rats in different groups ( $5 \mu \mathrm{m}$ thick sections, Cresyl violet staining, magnification: $\times 100$ ); Micrograph of the DG section in the sham groups (a), the normal number of neurons in the DG region. Micrograph of the DG section in nicotine group (b), decreased neurons cells due to the oxidative stress caused by nicotine. Micrograph of DG section in FV $(150 \mathrm{mg} / \mathrm{kg})$ group (c), the normal number of neurons. Micrograph of DG section in nicotine + FV $(100 \mathrm{mg} / \mathrm{kg})$ (d) and nicotine + FV $(150 \mathrm{mg} / \mathrm{kg}$ ) (e) groups (d), normal number of neurons. FV: Falcaria vulgaris, DG: Dentate gyrus.

of apoptosis and neurodegeneration by administering different doses of FV. The results of Tewari et al. were consistent with those of the present study that showed nicotine could damage the cells in the hippocampus by increased protein accumulation in the membrane and reduced cell size. ${ }^{19}$ Similarly, based on the results of the current study, nicotine could decrease significantly the number of neurons in the hippocampus due to damaging the cells. It seems that nicotine induces oxidative stress and, consequently, the production of free radicals such as superoxide and hydroxyl radicals, which can cause cell damage. ${ }^{10}$ Generated free radicals following oxidative stress may have the potential to damage cellular compositions, including proteins, lipids and DNA. ${ }^{16}$ The lipid in the membrane of the nerve cells has a high content of oxidized unsaturated fatty acids. Therefore, it seems that nicotine can produce reactive oxygen species via P-450 enzyme and cause the destruction of the nucleus in neurons. ${ }^{20}$ Equally, in the current study, nicotine could decrease the number of neurons and dendritic thorns in the hippocampus due to oxidative stress caused by administration of nicotine. Dendritic thorns play a major role in synaptic transmission. In this regard, many brain diseases are associated with changes in the morphology and density of dendritic thorns. ${ }^{15}$ Nicotine can reduce the length and the number of dendritic thorns in nucleus Accumbens by affecting the neurotrophic factors in the striatum. ${ }^{21} \mathrm{~A}$ study by Brown et al. showed that nicotine injections could reduce the length and the number of dendritic thorns; that is consistent with the results of our study. ${ }^{22}$ It seems that nicotine can destroy dendritic thorns by $\beta 2$-nAChRs deactivation at postsynaptic cells in hippocampus region. ${ }^{23}$ Moreover, nicotine can reduce the number of thorns by deactivating $\alpha 4 \beta 2$-nAChRs in the pre-synaptic membrane and by disrupting the release of glutamate neurotransmitters. ${ }^{24} \mathrm{FV}$ is a purifier of reactive oxygen species and has the potential to destroy oxidative stress and prevent lipid peroxidation. ${ }^{2}$ The results of the study by Rafiey et al. confirmed the results of the present study that FV could prevent cell death and development oxidative stress due to STZ administration. ${ }^{25}$ FV seems to inhibit lipid peroxidation of quinolinic acid and control the production of cyanide-induced superoxide. ${ }^{4} \mathrm{FV}$ extract can increase the effects of antioxidant enzymes such as catalase and superoxide dismutase and reduce ROS production. ${ }^{16}$ The present study showed a significant increase in serum nitrite oxide level in the nicotine group compared to the sham group. In all nicotine + FV groups, there was a significant decrease 


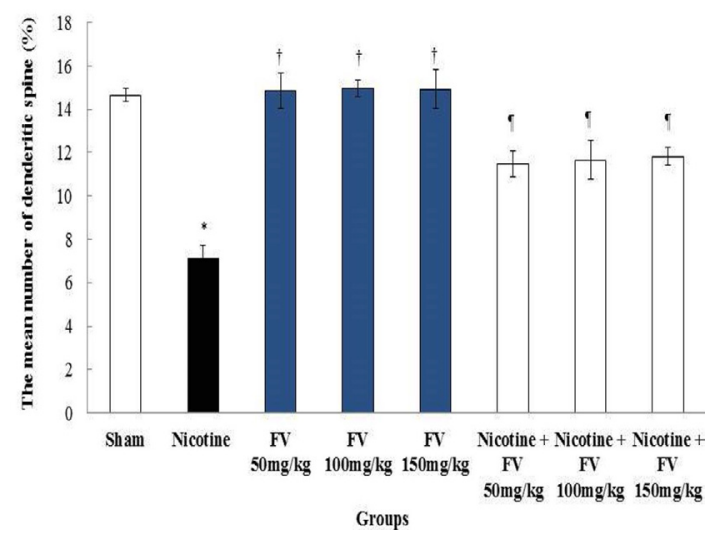

Figure 3: Comparison of nicotine, saline and FV groups at the mean number of dendritic spines in hippocampal region DG; *Significant difference compared to the sham group $(P<0.01)$. †Significant difference compared to the nicotine group $(P<0.01)$. ISignificant difference compared to the nicotine and sham groups $(P<0.01)$. FV: Falcaria vulgaris, DG: Dentate gyrus.

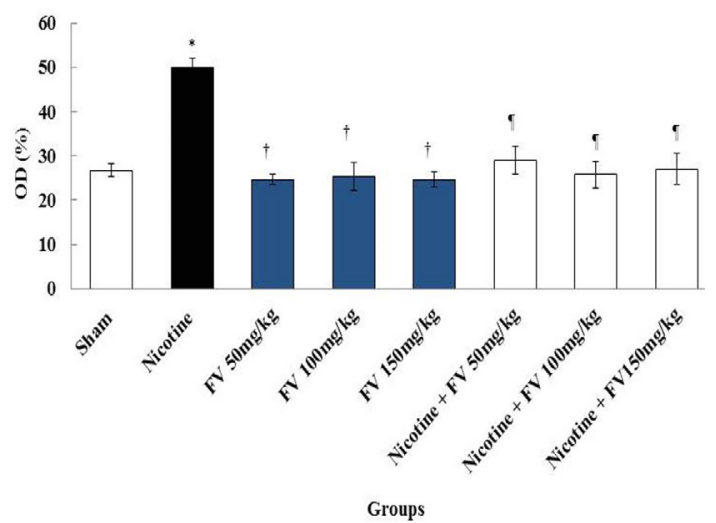

Figure 4: Microscopic images of neuronal dendritic spines in hippocampal region DG in male rats in different groups (Five-micron thick sections, Golgi staining, magnification: $\times 100$ ). Micrographs of the DG section in the sham group (a), normal structure. Micrograph of the DG section in nicotine group (b), decreased number of dendritic spines due to the oxidative stress caused by nicotine. Micrograph of the DG section in FV (100 mg/kg) group (c), normal structure; Micrograph of DG section in nicotine + FV (100 mg/kg) group (d) and $(150 \mathrm{mg} / \mathrm{kg}$ ) (e) groups, normal structure. FV: Falcaria vulgaris, DG: Dentate gyrus.

in serum nitrite oxide level in comparison to the nicotine group. Nitric oxide is a free radical and can regulate angiogenesis, apoptosis, cell cycle, invasion and metastasis in cells. ${ }^{26}$ Nicotine can stimulate nicotinic receptors in the brain and increase glutamate release and NMDA activation. The activation of NMDA may increase the formation of nitric oxide in the hippocampus. ${ }^{27}$ The results of a study by Keser et al. showed that exposure to nicotine increases the activity of nitric oxide in the frontal cortex in the mouse brain; consistent with the results of the present study. ${ }^{28}$ The results of this study showed that there was a significant decrease in total antioxidant levels in the nicotine group compared to the sham group. In all nicotine + FV groups, there was a significant decrease in serum total antioxidant levels in comparison to the nicotine group. Kahnamoei et al. showed that total antioxidant level was significantly reduced in smokers in proportion to non-smokers; which is consistent with the results of the current study. ${ }^{29}$ The reduction in total antioxidant capacity level in this study shows the effects of oxidative stress from nicotine on the hippocampal neuron. Nicotine induces oxidative stress in neural tissue that is demonstrated as a growth in the levels of ROS and lipid

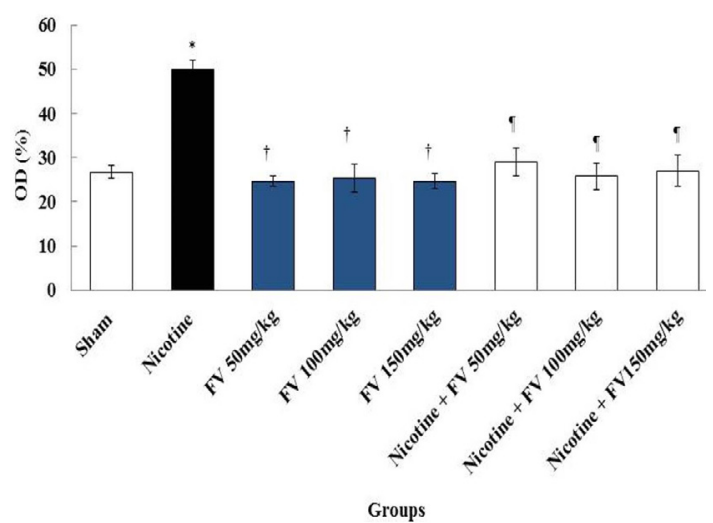

Figure 5: Effects of FV, nicotine and nicotine + FV on the mean of the nitrite oxide levels; ${ }^{*}$ Significant difference compared to the sham group $(P<0.01)$. †Significant difference compared to the nicotine group $(P<0.01)$. ISignificant difference compared to the nicotine group $(P<0.01)$. FV: Falcaria vulgaris, DG: Dentate gyrus.

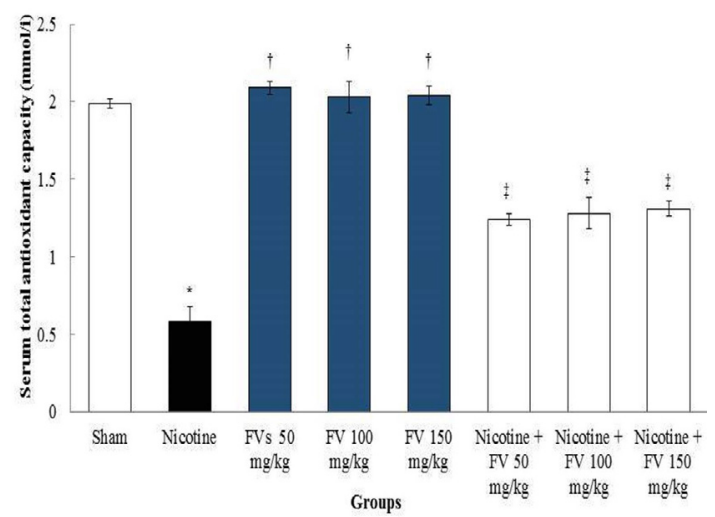

Figure 6: Comparison of total antioxidant capacity in nicotine and sham and $\mathrm{FV}$ and nicotine + FV groups; ${ }^{*}$ Significant difference compared to the sham group $(P<0.01)$. + Significant difference compared to the nicotine group $(P$ $<0.01)$. $\neq$ Significant difference compared to the nicotine group $(P<0.01)$. FV: Falcaria vulgaris, DG: Dentate gyrus.

peroxidation and a reduction in the action of antioxidant enzymes like total antioxidant capacity. ${ }^{30}$ In the present study, improved levels of total antioxidant capacity in rats treated with FV highlight the antioxidant effects of FV. ${ }^{3}$ A total antioxidant level increase due to the administration of nicotine indicates the positive impact of FV on magnified antioxidant effects and also the inhibition of nicotine-induced inflammation and destruction process on neurons in the brain. Further, it is assumed that Saponins in the extract of FV inhibit the synthesis of nitric oxide induction enzyme (iNOS). ${ }^{4}$ The results of the study by Jalili et al. are consistent with the results of the present study, which indicated that FV administration could inhibit nitric oxide production. ${ }^{4}$ The results of the present study showed that FV administration in all doses studied might have a positive effect on nicotine-induced toxicity, as an oxidative stress, in the neurons of the DG region and these effects were not associated with an increase in the dose of Falcaria extract.

\section{CONCLUSION}

It appears that FV provides protection against oxidative stress resulting from nicotine in the DG region. Such an ability of the FV might be due to its strong potential antioxidant attributes. FV administration moderates the antioxidant agents in the extract. As a result, it leads to DG tissue recovery and prevention of nicotine adverse effects on total antioxidant 
capacity, nitric oxide, number of neurons and dendritic spines as evidenced in the male rats.

\section{ACKNOWLEDGEMENT}

We gratefully acknowledge the Research Council of Kermanshah University of Medical Sciences (no: 97601) for the financial support.

\section{CONFLICT OF INTEREST}

The authors declare no conflict of interest.

\section{ABBREVIATIONS}

DG: Dentate gyrus; FV: Falcaria vulgaris; FRAP: Ferric reducing antioxidant power; AChRs: Acetylcholine receptors; OD: Optical density.

\section{REFERENCES}

1. Salahshoor MR, Mohammadi MM, Roshankhah S, Najari N, Jalili C. Effect of Falcaria vulgaris on oxidative damage of liver in diabetic rats. J Diabetes Metab Disord. 2019;18(1):15-23.

2. Rafiey Z, Jalili F, Sohrabi M, Salahshoor MR, Jalili C. Effects of hydro-alcoholic extract of Falcaria vulgaris on pancreas tissue in streptozotocin-induced diabetic rats. Iranian J Endocrin Metab. 2017;19(2):91-8.

3. Hamideh J, Khosro P, Javad ND. Callus induction and plant regeneration from leaf explants of Falcaria vulgaris an important medicinal plant. J Med Plant Res. 2012;6(18):3407-14

4. Jalili C, Kamani M, Roshankhah S, Sadeghi H, Salahshoor MR. Effect of Falcaria vulgaris extracts on sperm parameters in diabetic rats. Androl. 2018;50(10):e13130.

5. Fazly BBS, Haririzadeh G, Imami SA, Rashed MH. Survey of Iranian plants for alkaloids, flavonoids, saponins and tannins [Khorasan Province]. Int J Pharm. 1997;35(1):17-30.

6. Esfandiari E, Roshankhah S, Mardani M, Hashemibeni B, Naghsh E, Kazemi M, et al. The effect of high frequency electric field on enhancement of chondrogenesis in human adipose-derived stem cells. Iran J Basic Med Sci. 2014;17(8):571 6.

7. Kozlowski LT, Mehta NY, Sweeney CT, Schwartz SS, Vogler GP, Jarvis MJ, et al. Filter ventilation and nicotine content of tobacco in cigarettes from Canada, the United Kingdom and the United States. Tob Control. 1998;7(4):369-75.

8. Filbey FM, Gohel S, Prashad S, Biswal BB. Differential associations of combined vs. isolated cannabis and nicotine on brain resting state networks. Brain Struct Func. 2018;7(7):1-10.

9. Timmerman $W$, Westerink $B H$. Brain microdialysis of GABA and glutamate: What does it signify?. Synapse. 1997;27(3):242-61.

10. Jalili C, Khani F, Salahshoor MR, Roshankhah SH. Protective effect of curcumin against nicotine-induced damage on reproductive parameters in male mice. Int J Morphol. 2014;32(3):844-9.

11. Decina P, Caracci G, Sandik R, Berman W, Mukherjee S, Scapicchio P. Cigarette smoking and neuroleptic-induced parkinsonism. Biol Psychiatry. 1990;28(6):502
12. Salahshoor MR, Mohamadian S, Kakabaraei S, Roshankhah SH, Jalili C. Curcumin Improves liver Damage in Male Mice Exposed to Nicotine. J Tradit Complement Med. 2016;6(2):176-83

13. Changeux JP. Nicotine addiction and nicotinic receptors: Lessons from genetically modified mice. Nature Rev Neurosci. 2010;11(6):389-95.

14. Jalili. C, Salahshoor MR, Pourmotabbed A, Moradi S, Motaghi M, Shabanizadeh Darehdori A, et al. The Effects of Aqueous Extract of Boswellia serrata on Hippocampal Region CA1 and learning deficit in kindled Rats. Res Pharm Sci. 2014;9(5):351-8.

15. Luna VM, Anacker C, Burghardt NS, Khandaker H, Andreu V, Millette A, et al. Adult-born hippocampal neurons bidirectionally modulate entorhinal inputs into the dentate gyrus. Science. 2019;364(6440):578-83.

16. Jalili C, Ahmadi S, Roshankhah S, Salahshoor M. Effect of Genistein on reproductive parameter and serum nitric oxide levels in morphine-treated mice. Int J Reprod Biomed. 2016;14(2):95-102.

17. Benzie IF, Strain JJ. The Ferric Reducing Ability of Plasma (FRAP) as a measure of "antioxidant power": the FRAP assay. Anal Biochem. 1996;239(1):70-6.

18. Huhn S, Beyer F, Zhang R, Lampe L, Grothe J, Kratzsch J, et al. Effects of resveratrol on memory performance, hippocampus connectivity and microstructure in older adults-A randomized controlled trial. Neurolmage. 2018;174:177-90.

19. Salahshoor MR, Roshankhah S, Hosseni P, Jalili C. Genistein Improves Liver Damage in Male Mice Exposed to Morphine. Chin Med J. 2018;131(13):1598604.

20. Oddo S, Caccamo A, Green KN, Liang K, Tran L, Chen Y, et al. Chronic nicotine administration exacerbates tau pathology in a transgenic model of Alzheimer's disease. Proc Natl Acad Sci USA. 2005;102(8):3046-51.

21. Androuin A, Potier B, Nägerl UV, Cattaert D, Danglot $L$, Thierry M, et al. Evidence for altered dendritic spine compartmentalization in Alzheimer's disease and functional effects in a mouse model. Acta Neuropathol. 2018:25:1-6.

22. Brown R. Nicotine sensitization increases dendritic length and spine density in the nucleus accumbens and cingulate cortex. Brain Res. 2001;899(1-2):94-100

23. Blum R, Kafitz KW, Konnerth A. Neurotrophin-evoked depolarization requires the sodium channel $\mathrm{Na}(\mathrm{V}) 1.9$. Nature. 2002;419(6908):687-93.

24. Oda A, Nakagomi S. Nicotine induces dendritic spine remodeling in cultured hippocampel neurons. J Neurochem. 2014;128(2):246-55.

25. Shin HJ, Son E, Lee DH, Kim HJ, Kang SS, Cho GJ, et al Curcumin attenuate the Kainic acid Induced hippocampal cell death in mice. Neurosci Lett. 2007;416(1):49-54.

26. Ying L, Hofseth LJ. An emerging role for endothelial nitric oxide synthase in chronic inflammation and cancer. Cancer Res. 2007;67(4):1407-10.

27. Piri M, Nasehi M, Shahab Z, Zarrindast MR. The effects of nicotine on nitric oxide induced anxiogenic-like behaviors in the dorsal hippocampus. Neurosci Lett. 2012;528(2):93-8

28. Keser A, Nesil T, Kanit L, Pogun S. Brain nitric oxide metabolites in rats preselected for nicotine preference and intake. Neurosci Lett. 2013;545:102-6.

29. Kahnamoei JR, Maleki F, Nasirzadeh M, Kishizadeh F. The Effects of Cigarette Smoking on Plasma MDA And TAC in University Students. Bull Env Pharmacol Life Sci. 2014:3:95-8.

30. Nickander KK, Mcphee BR, Low PA, Tritschler H. Alpha-lipoic acid: Antioxidant potency against lipid peroxidation of neural tissues in vitro and implications for diabetic neuropathy. Free Radic Biol Med. 1996;21(5):631-9. 